\section{Prevalência e fatores associados para sobrepeso e obesidade em escolares de uma cidade no Sul do Brasil}

\author{
Prevalence of overweight and obesity and \\ associated factors among schoolchildren \\ in a southern Brazilian city
}

\author{
${ }^{1}$ Faculdade de Educação \\ Física e Ciência do Desporto, \\ Pontifícia Universidade \\ Católica do Rio Grande do \\ Sul, Porto Alegre, Brasil. \\ 2 Programa de Pós-graduação \\ em Saúde Coletiva, \\ Universidade do Vale do \\ Rio dos Sinos, \\ São Leopoldo, Brasil. \\ 3 Faculdade de Medicina \\ Universidade Federal de \\ Pelotas, Pelotas, Brasil. \\ Correspondência \\ J. S. Dias-da-Costa \\ Faculdade de Medicina \\ Universidade Federal de \\ Pelotas. \\ Av. Duque de Caxias 250, \\ Pelotas, RS \\ 96030-002, Brasil. \\ jcosta@epidemio-ufpel.org.br
}

\begin{abstract}
This cross-sectional study aimed to determine the prevalence of overweight and obesity in a group of 11-13-year-old schoolchildren in Capão da Canoa, Rio Grande do Sul State, Brazil, and to investigate the association with possible risk factors for excess body weight. The sample was stratified, proportional to the number of pupils in each school. Nutritional status was assessed by body mass index (BMI) cutoffs according to age and gender as proposed by Cole et al. (2000). Socioeconomic, demographic, genetic, and behavior variables were analyzed. 719 children from 11 schools were interviewed, of whom there were 541 (75.2\%) with normal BMI, 153 (21.3\%) with overweight, and 25 (3.5\%) with obesity. Statistically significant associations were found with type of school, parents' nutritional status, level of physical activity, and time of sedentary behavior. Prevalence of overweight and obesity in these schoolchildren was high (24.8\%), in keeping with the literature, confirming the problem's magnitude and severity in Brazil.
\end{abstract}

Obesity; Nutritional Status; Exercise; Students; Cross-Sectional Studies
Fabio Rodrigo Suñé 1,2

Juvenal Soares Dias-da-Costa 2,3

Maria Teresa Anselmo Olinto 2

Marcos Pascoal Pattussi 2

\section{Introdução}

Dados da Organização Mundial da Saúde (OMS) têm revelado uma proporção crescente de adultos com sobrepeso e obesidade, cerca de $50 \%$ dos adultos dos Estados Unidos, Canadá e de alguns países da Europa Ocidental apresentam índice de massa corporal (IMC), obtido através da equação (peso em kg/altura em $\mathrm{m}^{2}$ ), superior a $25 \mathrm{~kg} / \mathrm{m}^{2}$, e em alguns subgrupos a prevalência de sobrepeso é superior a $70 \%{ }^{1}$. No Brasil, a prevalência de obesidade em adultos também pode ser considerada um problema de saúde pública. Um estudo de base populacional realizado numa cidade do sul do Brasil, incluindo a população adulta, revelou uma prevalência de $21 \%$ entre homens e mulheres 2. Mais recentemente, a Pesquisa de Orçamentos Familiares (POF 2002-2003), revelou uma prevalência de sobrepeso e obesidade de $41,1 \%$ entre os homens e $40 \%$ entre as mulheres, acima de 20 anos 3 . Também na POF 2002-2003 o excesso de peso nos indivíduos de 12 e 14 anos do sexo masculino foi de $18,4 \%$, e de $16,6 \%$ no sexo feminino 4 .

Além disso, de acordo com a OMS estudos têm revelado aumento da prevalência de obesidade na infância e adolescência independentemente do método de classificação antropométrico utilizado. Nos Estados Unidos, a prevalência de sobrepeso (definida por medidas superiores ao percentil 85 do IMC) em indivíduos com idade entre 5 e 24 anos aproximadamente dobrou 
entre 1973 e 1994. Além disso, o aumento anual no peso relativo e de obesidade foi $50 \%$ maior entre 1983 e 1994 comparado ao período entre 1973 e 1982. Uma tendência similar tem sido observada no Japão, onde a prevalência de escolares obesos entre 6 e 14 anos aumentou de 5\% para 10\% entre 1974 e 19931.

Estudos no Brasil reforçam as evidências apresentadas pela OMS. De acordo com Albano \& Souza 5 , a prevalência de sobrepeso ou obesidade alcançou $32,5 \%$ em meninos e $26,5 \%$ em meninas de uma escola pública de São Paulo. Souza-Leão et al. 6 encontraram uma prevalência de obesidade (IMC > p 95) de 15,8\% em escolares de Salvador, Bahia. Outros estudos representativos da população brasileira compararam tendências e mostraram aumento nas prevalências de sobrepeso. Wang et al. 7 demonstraram que a prevalência de sobrepeso era de 4,1\% em 1974, atingindo 13,9\% em 1997. Já Veiga et al. ${ }^{8}$ revelaram entre indivíduos de 10 a 19 anos um crescimento da prevalência de sobrepeso de 2,6\% em 1975 para $11,8 \%$ em 1997.

Sabe-se que existe uma associação entre a obesidade e diversas doenças crônicas não transmissíveis, tais como, doença coronariana, hipertensão, diabetes, dislipidemias sangüíneas, osteoartrite, entre outras ${ }^{9}$. Segundo dados do Departamento de Informática do SUS (DATASUS) 10, no Estado do Rio Grande do Sul, $38 \%$ dos óbitos no ano 2000 foram por doenças associadas à obesidade, quase $9 \%$ da população de 30 a 69 anos têm diabetes e, cerca de $17,7 \%$ das intervenções hospitalares na mesma faixa etária são por doenças do aparelho circulatório.

A obesidade infantil pode apresentar-se acompanhada também de transtornos psicossociais. Segundo Burrows 11, cerca de 50\% dos obesos apresentam menor sociabilidade, menor rendimento escolar, baixa auto-estima, além de distúrbios de humor e sono.

A obesidade pode ser causada por diversos fatores. De acordo com Wright et al. 12, crianças obesas filhas de pais obesos têm maiores chances de tornarem-se adultos obesos, devido a influências genéticas e também devido aos hábitos adotados pela família. Além disso, alguns estudos têm mostrado que o comportamento alimentar de adolescentes parece apresentar elevado consumo de lipídeos. Segundo Kazapi et al. 13, quase $34 \%$ dos alunos de escolas públicas e $39 \%$ dos alunos de escolas particulares apresentam consumo elevado deste nutriente.

Os longos períodos de sedentarismo também podem ser responsabilizados pelo acúmulo de gordura corporal. Dados apontam para uma associação positiva entre sobrepeso e horas de conduta sedentária 14, ou ainda não assistir à te- levisão ou assistir menos de 2 horas por dia, comparado a quem assiste por mais de 5 horas por dia, está associado positivamente à realização de alguma atividade física de lazer ${ }^{15}$.

Outro fator que pode estar associado ao aumento na prevalência de sobrepeso e obesidade é o nível de atividade física diária realizada por crianças e adolescentes. Estudo realizado em Niterói, Rio de Janeiro, entre 1997 e 1998, apresentou prevalências de sedentarismo de $85 \%$ em meninos e $94 \%$ em meninas com idade entre 10 e 19 anos 16 .

Assim, este estudo foi realizado com o objetivo de determinar a prevalência de obesidade entre escolares, bem como investigar sua associação com possíveis fatores que possam favorecer o excesso de peso corporal.

\section{Materiais e métodos}

Foi realizado um estudo transversal incluindo amostra de escolares de ambos os sexos, de 11 a 13 anos de idade, regularmente matriculadas nas escolas do Município de Capão da Canoa, Rio Grande do Sul, Brasil, durante o ano de 2004.

O Município de Capão da Canoa situa-se no litoral norte do Rio Grande do Sul e fica distante cerca de $140 \mathrm{~km}$ da capital do Estado. A população do município no ano de 2003 era de 33.891 habitantes, e destes, 2.059 tinham idade compreendida entre 11 e 13 anos. Capão da Canoa conta com dez escolas municipais, três escolas estaduais e duas escolas particulares com ensino fundamental. Entretanto, quatro das dez escolas municipais localizam-se nos distritos de Capão da Canoa.

A coleta de dados foi realizada no período compreendido entre os meses de novembro e dezembro de 2004, sendo que, as escolas localizadas nos distritos não foram incluídas no estudo.

O cálculo para determinar o tamanho da amostra baseou-se em uma prevalência estimada de $22 \%$ de sobrepeso e obesidade 2 , em um erro amostral de 3\%, um nível de confiança de 95\% para uma população de 1.682 escolares. Estimando-se, assim, que 510 escolares seriam suficientes para realizar o estudo de prevalência.

Entretanto, como os objetivos do estudo não se esgotavam na determinação de prevalência de sobrepeso e obesidade, e sim pela sua associação com o nível de atividade física diária e com o consumo de alimentos com alta densidade energética, foi necessário também o cálculo do tamanho da amostra para estas duas variáveis.

O cálculo do tamanho de amostra para o estudo de associação entre sobrepeso ou obesidade, atividade física e consumo de alimentos com 
alta densidade energética utilizou os seguintes parâmetros: freqüência de doença nos não expostos de $10 \%$; risco relativo de 2,0 , para as duas variáveis; poder de $80 \%$ e; nível de confiança de 95\%. Para o nível de atividade física foi considerada uma proporção de 1 ativo para cada 2 sedentários, conforme Souza-Leão et al. 6, e para o consumo de alimentos com alta densidade energética foi considerada uma proporção de 2 não expostos para cada 1 exposto, conforme Kazapi et al. 13 .

Sendo assim, estabeleceu-se como necessária uma amostra de 463 escolares para determinar associação com o nível de atividade física, e de 474 escolares para verificar associação com o consumo de alimentos com alta densidade energética.

Portanto, o maior tamanho de amostra para o estudo da prevalência de sobrepeso ou obesidade foi de 510 escolares. Acrescentou-se a esse número um efeito de desenho de 1,4, e uma previsão de $24 \%$ de perdas ou recusas de pais ou escolares, resultando em uma amostra final de 885 adolescentes. A elevada previsão de perdas e recusas se justificou em virtude de que a coleta dos dados ocorreria no final do ano letivo quando a evasão é maior, além do resultado do estudo piloto, que apresentou perdas e recusas ao redor de $20 \%$.

O processo de amostragem se deu utilizandose a técnica de multiestágio, primeiro se realizou uma amostragem estratificada proporcional ao número de alunos de cada escola, seguido de uma amostragem aleatória dentro de cada escola. Sendo que todas as escolas urbanas do município foram incluídas no estudo. Esse processo amostral permite que cada escolar de escola municipal, estadual ou particular tenha probabilidade igual de ser sorteado.

Após a realização de esclarecimentos acerca do trabalho a ser realizado junto à direção das escolas, aos pais e aos próprios adolescentes selecionados, a coleta de dados foi realizada, pelo próprio pesquisador, para evitar a variação interobservador. Foi realizado estudo piloto em escola de outro município objetivando padronizar a coleta dos dados.

Foram coletadas informações sobre idade, sexo, tipo de escola, escolaridade dos pais e tempo gasto em conduta sedentária, que compreende atividades como: assistir à televisão ou vídeo, jogar video-game e utilizar o computador, onde o gasto energético é nulo ou mínimo. A variável tempo em conduta sedentária foi dicotomizada, tendo ponto de corte 4 horas e 30 minutos 16 .

Como tentativa de caracterizar a percepção do estado nutricional dos pais foram criadas nove figuras, tentando-se utilizar a imagem visual da criança em relação aos pais. Aqueles apontados com número de figura superior a seis foram considerados com peso excessivo.

O consumo de alimentos de alta densidade energética foi verificado por meio de um conjunto simplificado de questões pela aceitação das crianças em respondê-lo e por questões logísticas de tempo de aplicação de questionários. O instrumento proposto por Chiara \& Sichieri 17 avalia o consumo de produtos alimentares marcadores de risco cardiovascular. Assim, o consumo pode ser classificado como adequado, alto consumo e consumo excessivo.

Os dados sobre o nível de atividade física foram coletados utilizando-se de um questionário de atividades físicas habituais, desenvolvido originalmente por Russell R. Pate da University of South Carolina (Estados Unidos), traduzido e modificado por M. V. Nahas do Núcleo de Pesquisa em Atividade Física e Saúde da Universidade Federal de Santa Catarina, que classifica o nível de atividades físicas habituais em inativo (0 a 5 pontos), moderadamente ativo (6 a 11 pontos), ativo (12 a 20 pontos) e muito ativo (21 ou mais pontos) 18.

O peso foi verificado duas vezes, utilizandose a mesma balança mecânica Filizola, modelo 31 (São Paulo, Brasil), com precisão de cem gramas $(0,1 \mathrm{~kg})$ e a altura avaliada usando-se um antropômetro com precisão decimal, também medida duas vezes. Para análise foram utilizadas as médias das medidas de peso e altura. A balança foi aferida a cada pesagem, por meio de peso padronizado.

A prevalência de sobrepeso e obesidade foi verificada através do IMC, utilizando pontos de corte para classificação de sobrepeso e de obesidade, de acordo com sexo e idade, estabelecidos por Cole et al. 19 .

Foi realizada dupla entrada de dados, para identificação de possíveis erros de digitação, através do programa Epi Info (Centers for Disease Control and Prevention, Atlanta, Estados Unidos).

A análise dos dados foi realizada através dos programas SPSS (SPSS Inc., Chicago, Estados Unidos) e Stata (Stata Corporation, College Station, Estados Unidos). Em um primeiro momento foi realizada uma analise univariada, para verificar as prevalências em cada um dos percentis utilizados como ponto de corte do desfecho (sobrepeso e obesidade), com os respectivos intervalos de confiança.

Os indivíduos classificados com sobrepeso foram agrupados aos obesos para análise bivariada, demonstrando-se as razões de prevalência, os intervalos de confiança de 95\% (IC95\%) e os resultados do teste de qui-quadrado e de 
tendência linear para as variáveis ordinais 20 . Por fim, para controle dos fatores de confusão, foi realizada regressão de Poisson, seguindo modelo hierarquizado 21 . O modelo hierárquico apresentado para este estudo foi montado após a revisão de literatura, e apresenta a relação entre as principais variáveis propostas para a análise (Figura 1). Assim, as variáveis sócio-econômicas, demográficas e de percepção do estado nutricional dos pais estavam num nível mais distal determinando as variáveis classificadas como comportamentais e todas associadas com o desfecho. Foram incluídas no modelo final as variáveis que apresentaram no teste de Wald pelo menos uma de suas categorias com valor menor que 0,05 .

A pesquisa foi aprovada no Comitê de Ética e Pesquisa da Universidade do Vale do Rio dos Sinos. A Secretaria Municipal de Educação de Capão da Canoa e as escolas envolvidas acordaram em participar e os pais das crianças autorizaram a participação dos alunos.

\section{Resultados}

Ao final da coleta de dados, devido em grande parte ao número de evasões escolares, chegou-se ao total de $18,7 \%$ de perdas, sendo que, em nenhum dos estratos este número superou o valor estimado no cálculo amostral.

Foram entrevistados 719 escolares em 11 escolas do Município de Capão da Canoa, destas, $541(75,2 \%)$ apresentaram IMC considerado normal. Entretanto, 153 escolares apresentaram IMC com valores superiores ao estabelecido como normal para indivíduos eutróficos e inferiores ao ponto de corte para obesidade, o que representa uma prevalência de sobrepeso de 21,3\% (IC95\%: 18,3-24,3). Outros 25 escolares apresentaram IMC igual ou superior ao ponto de corte adotado para a obesidade, caracterizando uma prevalência de 3,5\% (IC95\%: 2,1-4,8).

Para a seqüência das análises os indivíduos com sobrepeso foram agrupados aos obesos, totalizando então uma prevalência de 24,8\% (178) de indivíduos com sobrepeso ou obesidade.

No que se refere às variáveis sócio-econômicas, somente o tipo de escola apresentou diferença significativa $(p=0,001)$ entre suas categorias. Encontrou-se uma prevalência de sobrepeso e obesidade de $25,7 \%$ nas escolas municipais, $17,1 \%$ nas escolas estaduais e de $39,2 \%$ nas particulares. Além disso, alunos matriculados nas escolas estaduais apresentaram risco $33 \%$ menor de apresentar sobrepeso ou obesidade que os alunos matriculados em escolas municipais. Os alunos matriculados em escolas particulares têm risco 53\% maior de apresentar sobrepeso ou obesidade que os alunos das escolas municipais. As variáveis escolaridade do pai e escolaridade da mãe, não apresentaram diferenças estatisticamente significativas (Tabela 1).

A prevalência de sobrepeso e obesidade foi menor entre as meninas. Contudo, o nível superior do intervalo de confiança foi igual à unidade (Tabela 1). Em relação à idade, observou-se que os escolares de 13 anos apresentaram prevalência menor de sobrepeso ou obesidade do que os mais jovens, confirmada pelos intervalos de confiança (Tabela 1).

A prevalência de sobrepeso ou obesidade aumentou de acordo com o estado nutricional dos pais percebido pelos escolares incluídos no estudo, de acordo com os intervalos de confiança de 95\% e teste estatístico. Na análise foi encontrada associação linear significativa, de forma que os adolescentes com pelo menos um dos pais acima do peso apresentaram cerca de $50 \%$ de risco de apresentarem mais sobrepeso ou obesidade. Aquelas com ambos acima do peso mostravam o dobro de sobrepeso ou obesidade (Tabela 1).

Quanto às variáveis comportamentais, foi constatada uma prevalência de sobrepeso ou obesidade de $31,4 \%$ em escolares que permanecem mais de 4 horas e 30 minutos em conduta sedentária (Tabela 2).

$\mathrm{Na}$ avaliação do nível de atividade física dos alunos encontrou-se uma prevalência de sobrepeso ou obesidade de $7,8 \%$ nos muito ativos, de $13,4 \%$ nos ativos, de $48,2 \%$ nos moderadamente ativos e de $35,9 \%$ nos inativos. Foi observada a presença de teste de tendência linear com diferenças estatisticamente significativas. Quando o nível de atividade física foi classificado como moderadamente ativo e ativo, observou-se diferença significativa $(\mathrm{p}<0,0001)$ em relação à categoria de base. Sendo que, com um aumento superior a seis vezes no risco de apresentar sobrepeso ou obesidade em adolescentes moderadamente ativos e superior a quatro vezes no risco de sobrepeso e obesidade nos adolescentes inativos.

A análise da variável sobre consumo de alimentos com alta densidade energética apontou para diferenças estatisticamente significativas. Os escolares com consumo classificado como alto, apresentaram mais da metade do risco de apresentar sobrepeso ou obesidade. Já o consumo excessivo não apresentou associação com sobrepeso ou obesidade, pois o intervalo de confiança incluiu a unidade (Tabela 2).

Os resultados da regressão de Poisson mostraram associações estatisticamente significativas para as variáveis: tipo de escola, idade, estado nutricional dos pais, tempo de conduta sedentária e nível de atividade física (Tabela 3). 


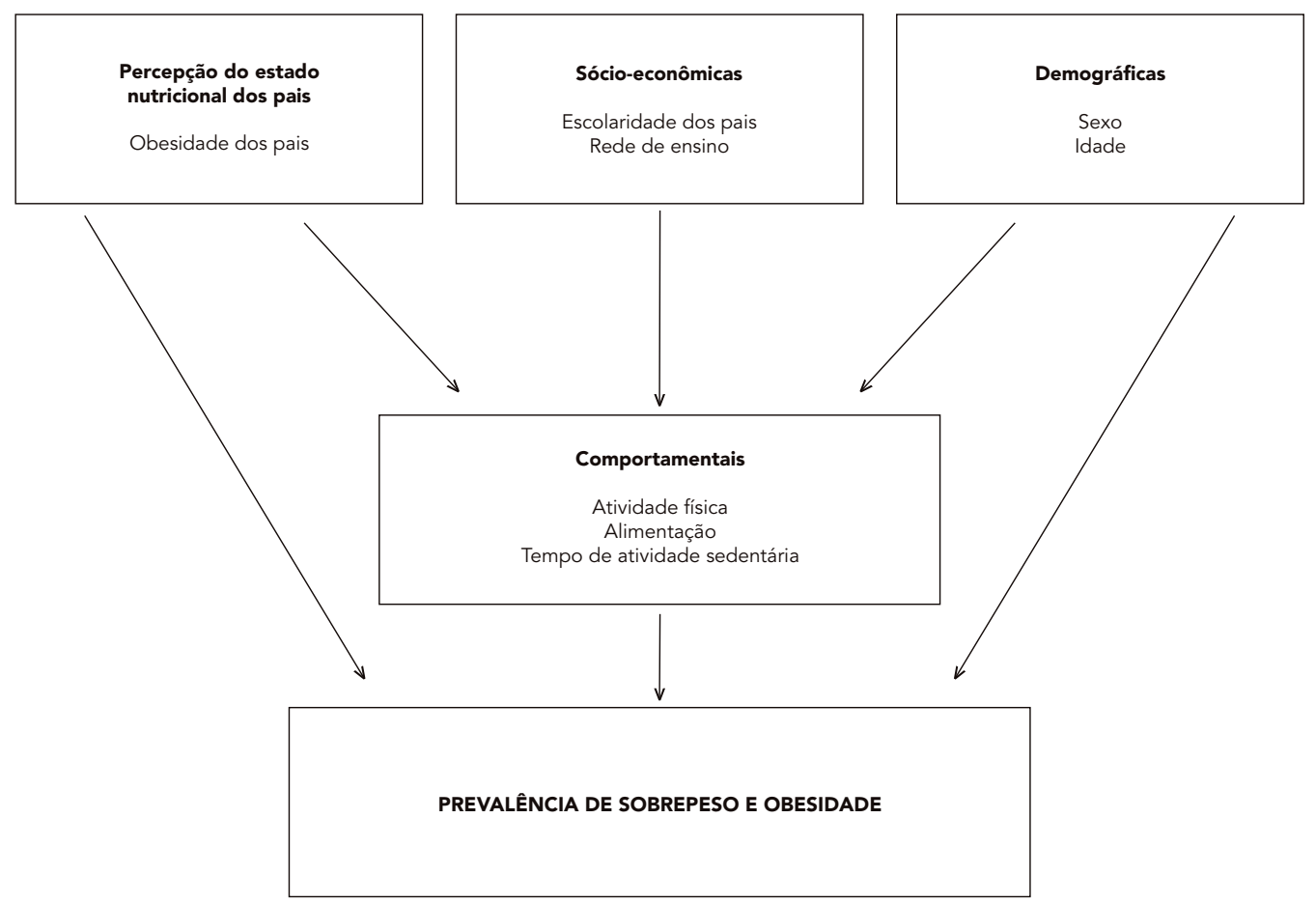

\section{Discussão}

Uma das limitações encontradas neste estudo foi o grande número de perdas devido à evasão escolar. Entretanto, esse número apresentou-se homogêneo entre as escolas, incluindo as de natureza privada, não superando o valor previsto na amostra em nenhum dos estratos, diminuindo assim sua interferência nos resultados do estudo.

A coleta dos dados foi realizada no período compreendido entre a última quinzena de novembro e a primeira de dezembro, época em que as escolas totalizam seus índices de evasão e abandono. Assim, a limitação por evasão pode ser explicada parcialmente pelas características sazonais de emprego e renda, apresentadas pela região litorânea do estado, na época do ano em que o estudo foi realizado, bem como pela proximidade com o término do ano letivo, momento em que os alunos que já atingiram os objetivos da série, têm a freqüência escolar reduzida.

A falta de validação do instrumento para coleta de informações acerca da prática de ativida- de física constituiu-se como uma outra limitação do estudo, contudo, durante a revisão da literatura não foram encontrados instrumentos devidamente validados para a população brasileira nesta faixa etária.

A prevalência de sobrepeso e obesidade encontrada nos escolares avaliados mostrou-se elevada $(24,8 \%)$, e, de acordo com a encontrada na literatura, independente do critério de diagnóstico utilizado, confirmando a magnitude e gravidade que o problema assumiu entre os escolares de todo o Brasil. Nos estudos que serviram de comparação foram encontradas prevalências que variaram de $18,8 \%$ a $32,5 \%$ em meninos, e de $21,1 \%$ a $30,9 \%$ em meninas $5,22,23,24$. Deve ser dada a devida atenção às diferenças nas prevalências advindas de diferenças na composição corporal por sexo e grupo etário, nos estudos utilizados para comparação.

Dentre as variáveis propostas no estudo para explorar a associação de sobrepeso ou obesidade com as características sócio-econômicas, a única que apresentou associação significativa foi o tipo de escola, onde se pode observar que 
Prevalência de sobrepeso ou obesidade em escolares de 11 a 13 anos, de acordo com as variáveis sócio-econômicas, demográficas e estado nutricional dos pais. Capão da Canoa, Rio Grande do Sul, Brasil, 2004.

\begin{tabular}{|c|c|c|c|c|c|}
\hline Variável & $\mathrm{n}$ & $\begin{array}{c}\text { Prevalência de sobrepeso } \\
\text { ou obesidade }\end{array}$ & $\begin{array}{l}\text { Razão de } \\
\text { prevalência }\end{array}$ & $\begin{array}{c}\text { Intervalo de } \\
\text { confiança de } 95 \%\end{array}$ & $\mathrm{p}$-valor \\
\hline Tipo de escola & & & & & 0,001 \\
\hline Municipal & 452 & $116(25,7 \%)$ & 1,00 & & \\
\hline Estadual & 193 & $33(17,1 \%)$ & 0,67 & $0,47-0,94$ & \\
\hline Particular & 74 & $29(39,2 \%)$ & 1,53 & $1,10-2,11$ & \\
\hline Escolaridade do pai (anos) & & & & & 0,28 \\
\hline 11 ou + & 146 & $35(24,0 \%)$ & 1,00 & & \\
\hline $8-10$ & 187 & $46(24,6 \%)$ & 1,03 & $0,70-1,50$ & \\
\hline $5-7$ & 233 & $68(29,2 \%)$ & 1,22 & $0,86-1,73$ & \\
\hline $0-4$ & 142 & $29(20,4 \%)$ & 0,85 & $0,55-1,32$ & \\
\hline Escolaridade da mãe (anos) & & & & & 0,85 \\
\hline 11 ou + & 164 & $44(26,8 \%)$ & 1,00 & & \\
\hline $8-10$ & 176 & $40(22,7 \%)$ & 0,85 & $0,58-1,23$ & \\
\hline $5-7$ & 242 & $61(25,2 \%)$ & 0,94 & $0,67-1,31$ & \\
\hline $0-4$ & 132 & $33(25,0 \%)$ & 0,93 & $0,63-1,37$ & \\
\hline Sexo & & & & & 0,049 \\
\hline Meninos & 358 & $100(27,9 \%)$ & 1,00 & & \\
\hline Meninas & 361 & $78(21,6 \%)$ & 0,77 & $0,60-1,00$ & \\
\hline Idade (anos) & & & & & 0,063 * \\
\hline 11 & 256 & $73(28,5 \%)$ & 1,00 & & \\
\hline 12 & 241 & $62(25,7 \%)$ & 0,90 & $0,68-1,20$ & \\
\hline 13 & 222 & $43(19,4 \%)$ & 0,68 & $0,49-0,95$ & \\
\hline Estado nutricional dos pais & & & & & $<0,001$ * \\
\hline Normal & 358 & $67(18,7 \%)$ & 1,00 & & \\
\hline Um acima do peso & 251 & $71(28,3 \%)$ & 1,51 & $1,13-2,02$ & \\
\hline Ambos acima do peso & 101 & $40(39,6 \%)$ & 2,12 & $1,53-2,92$ & \\
\hline
\end{tabular}

* Teste de tendência linear.

adolescentes matriculados na rede pública de ensino apresentaram menores prevalências de sobrepeso ou obesidade do que aqueles inseridas na rede particular de ensino. Resultado semelhante ao observado por Souza-Leão et al. 6, onde foi encontrada uma prevalência de $30 \%$ em escolas particulares e $8 \%$ em escolas públicas de Salvador, Bahia.

As variáveis sócio-econômicas mais freqüentemente utilizadas em estudos epidemiológicos, tais como renda familiar, renda familiar per capita ou classificação econômica da Associação Brasileira de Empresas de Pesquisa (ABEP; http://www.abep.org/) não foram coletadas nesta investigação em virtude dos respondentes serem os próprios escolares. Assim, as variáveis sócio-econômicas analisadas foram: escolaridade dos pais e tipo de escola, na suposição de que integrantes de escolas privadas tivessem uma in- serção de classe mais elevada do que os alunos das escolas públicas, por ser a variável uma proxi de classe social.

De qualquer forma, os achados referentes ao tipo de escola mostraram que a prevalência de sobrepeso ou obesidade era maior nos escolares da rede privada. Contudo, esperava-se que as crianças com melhores condições de vida apresentassem melhores resultados de saúde relacionados ao excesso de peso, uma vez que estão protegidas para desnutrição e doenças associadas à situação sócio-econômica 25,26.

Não foram encontradas diferenças significativas entre os sexos, resultado semelhante ao encontrado por Carvalho et al. 27 , quando avaliaram o consumo alimentar em adolescentes com idade entre 10 e 19 anos de uma escola particular de Teresina, Piauí, no ano de 2001, e deram indícios de que o acúmulo de gordura corporal indepen- 
Prevalência de sobrepeso ou obesidade em escolares de 11 a 13 anos, de acordo com as variáveis comportamentais. Capão da Canoa, Rio Grande do Sul, Brasil, 2004.

\begin{tabular}{|c|c|c|c|c|c|}
\hline Variável & $\mathrm{n}$ & $\begin{array}{c}\text { Prevalência de sobrepeso } \\
\text { ou obesidade }\end{array}$ & $\begin{array}{l}\text { Razão de } \\
\text { prevalência }\end{array}$ & $\begin{array}{c}\text { Intervalo de } \\
\text { confiança de } 95 \%\end{array}$ & p-valor \\
\hline Tempo de conduta sedentária & & & & & $<0,01$ \\
\hline Até 4 horas e 30 minutos & 493 & $107(21,7 \%)$ & 1,00 & & \\
\hline Mais de 4 horas e 30 minutos & 226 & $71(31,4 \%)$ & 1,45 & $1,12-1,87$ & \\
\hline Nível de atividade física & & & & & $<0,0001$ * \\
\hline Muito ativo & 102 & $9(7,8 \%)$ & 1,00 & & \\
\hline Ativo & 352 & $47(13,4 \%)$ & 1,70 & $0,83-3,49$ & \\
\hline Moderadamente ativo & 226 & $109(48,2 \%)$ & 6,15 & $3,12-12,12$ & \\
\hline Inativo & 39 & $14(35,9 \%)$ & 4,58 & $2,08-10,05$ & \\
\hline \multicolumn{6}{|l|}{ Consumo de alimentos } \\
\hline com alta densidade energética & & & & & $<0,0001$ \\
\hline Adequado & 226 & $53(23,5 \%)$ & 1,00 & & \\
\hline Alto & 133 & $51(38,6 \%)$ & 1,64 & $1,19-2,25$ & \\
\hline Excessivo & 360 & $74(20,6 \%)$ & 0,64 & $0,64-1,20$ & \\
\hline
\end{tabular}

* Teste de tendência linear.

de do sexo para esta faixa etária. Por outro lado, Fonseca et al. 14, num estudo que incluiu 391 indivíduos de 15 a 17 anos de uma escola privada em Niterói, mostraram uma maior prevalência de sobrepeso e obesidade nos meninos.

Em relação à idade, os estudantes com 13 anos mostraram-se protegidos quando comparados com aqueles de 11 anos. Esse achado talvez possa ser explicado pelas diferenças maturacionais que podem estar presentes nessa faixa etária, pois é por volta dos 13 anos, com o aumento da liberação dos hormônios sexuais, que se dá o estirão do crescimento 28 .

Encontrou-se associação significativa entre a prevalência de sobrepeso e obesidade e a percepção do estado nutricional dos pais. Assim, adolescentes, filhos de pais com estado nutricional considerado normal pela percepção possuíam menores probabilidades de apresentarem sobrepeso e obesidade do que aqueles com o pai ou a mãe acima do peso. A medida de efeito era ainda maior nos adolescentes com pai e mãe acima do peso, resultado em acordo com o apresentado por Ramos \& Barros Filho 29, em uma população com idade entre 11 e 18 anos da rede estadual de ensino de Bragança Paulista, onde a prevalência de sobrepeso ou obesidade entre adolescentes com pai e mãe obesos foi de $26,1 \%$ e de $9,3 \%$ entre aqueles com pai e mãe com peso normal. Deve-se considerar que a percepção da obesidade de pais e mães foi realizada por meio da identificação de figuras em instrumento ainda não validado.

Acompanhando os resultados já encontrados na literatura, onde Frutuoso et al. 30 detectaram associação significativa entre a realização de atividades passivas e a prevalência de sobrepeso e obesidade em adolescentes de 10 a 13 anos de idade, freqüentadores de um centro de juventude, no Município de São Paulo, e Fonseca et al. 14, que descreveram associação positiva e significativa entre a prevalência de sobrepeso e obesidade e o tempo em conduta sedentária, em escolares de classe média, com idade entre 15 e 17 anos de uma escola privada de Niterói, os dados de nosso estudo confirmaram a associação entre o tempo de conduta sedentária e a prevalência de sobrepeso ou obesidade. Adolescentes que permanecem em média mais de 4 horas e 30 minutos por dia em conduta sedentária têm maiores chances de apresentar sobrepeso ou obesidade. Além disso, a literatura já aponta para uma associação entre o tempo de conduta sedentária e a realização de atividades físicas de lazer 15 .

A associação do nível de atividade física com a prevalência de sobrepeso ou obesidade por meio de estudos transversais ainda apresenta-se controversa. O estudo de Fonseca et al. 14 investigou o nível de atividade física através de questões que englobavam o tipo, o tempo e a freqüência das atividades realizadas, não revelou associação significativa entre estas variáveis. Já Jenovesi et 
Análise multivariada para prevalência de sobrepeso ou obesidade em escolares de 11 a 13 anos, de acordo com as variáveis incluídas no modelo.

Capão da Canoa, Rio Grande do Sul, Brasil, 2004

\begin{tabular}{|c|c|c|c|}
\hline Variável & $\begin{array}{l}\text { Razão de } \\
\text { prevalência }\end{array}$ & $\begin{array}{c}\text { Intervalo de } \\
\text { confiança de } 95 \%\end{array}$ & $\mathrm{p}$ valor \\
\hline \multicolumn{4}{|l|}{ Tipo de escola } \\
\hline Municipal & 1,00 & & \\
\hline Estadual & 0,93 & $0,88-0,99$ & 0,02 \\
\hline Particular & 1,10 & $1,01-1,20$ & 0,03 \\
\hline \multicolumn{4}{|l|}{ Sexo } \\
\hline Meninos & 1,00 & & \\
\hline Meninas & 0,96 & $0,91-1,00$ & 0,08 \\
\hline \multicolumn{4}{|l|}{ Idade (anos) } \\
\hline 11 & 1,00 & & \\
\hline 12 & 0,97 & $0,91-1,03$ & 0,41 \\
\hline 13 & 0,92 & $0,87-0,98$ & 0,01 \\
\hline \multicolumn{4}{|l|}{ Estado nutricional dos pais } \\
\hline Normal & 1,00 & & \\
\hline Um acima do peso & 1,08 & $1,02-1,14$ & $<0,01$ \\
\hline Ambos acima do peso & 1,18 & $1,09-1,28$ & $<0,0001$ \\
\hline \multicolumn{4}{|l|}{ Tempo de conduta sedentária * } \\
\hline Até 4 horas e 30 minutos & 1,00 & & \\
\hline Mais de 4 horas e 30 minutos & 1,06 & $1,01-1,12$ & 0,02 \\
\hline \multicolumn{4}{|l|}{ Nível de atividade física * } \\
\hline Muito ativo & 1,00 & & \\
\hline Ativo & 1,04 & $0,98-1,10$ & 0,18 \\
\hline Moderadamente ativo & 1,33 & $1,25-1,43$ & $<0,0001$ \\
\hline Inativo & 1,19 & $1,06-1,35$ & $<0,01$ \\
\hline \multicolumn{4}{|c|}{ Consumo de alimentos com alta densidade energética * } \\
\hline Adequado & 1,00 & & \\
\hline Alto & 1,05 & $0,98-1,12$ & 0,13 \\
\hline Excessivo & 0,96 & $0,90-1,01$ & 0,1 \\
\hline
\end{tabular}

*Ajustados para tipo de escola, idade e estado nutricional dos pais.

al. 31 demonstraram a existência de associação significativa entre a prevalência de sobrepeso e obesidade e os níveis de atividade física, em escolares de escolas públicas, da 1ạ e 2a séries do ensino fundamental, da cidade de São Paulo, utilizando um questionário de autopreenchimento, respondido pelos pais.

Neste estudo o nível de atividade física diária mostrou estar significativamente associado à prevalência de sobrepeso e obesidade. Embora esse resultado deva ser avaliado com cautela, devido à falta de validação do instrumento de coleta de dados, ele pode ser utilizado como indicativo do perfil de atividade física assumida pela população em estudo.

A falta de instrumentos específicos para a população brasileira, as diferentes formas de coleta de dados e a enorme dificuldade de utilização de métodos diretos na avaliação do nível de atividade física, podem ser as responsáveis pela grande diferença nos resultados encontrados entre os estudos.

Estudos transversais com adultos têm mostrado uma relação de causalidade reversa entre atividade física e obesidade, entretanto, em estudos com crianças isso não é observado 31 . O presente estudo pode ter encontrado o ponto de quebra da causalidade reversa para essas variáveis. No momento em que a criança atinge a adolescência e a existência do problema passa a incomodar, as medidas necessárias para a sua solução são tomadas, entre elas, a inclusão de um programa de exercícios físicos na rotina. Entretanto, novos estudos com adolescentes devem 
ser realizados para clarear a relação entre essas duas variáveis.

Em um primeiro momento este estudo demonstrou associação significativa entre o alto consumo de alimentos com alta densidade energética e sobrepeso ou obesidade, entretanto, após a realização da regressão de Poisson foi perdida a significância estatística. Esse resultado pode estar mascarado devido ao viés de informação, pois aqueles sabidamente com excesso de peso podem manipular a informação a seu favor. Em alguns estudos 14,32 tem-se observado que o consumo de alimentos com alta densidade energética por indivíduos obesos é semelhante ou menor ao de indivíduos eutróficos, o que pode revelar uma tendência dos obesos de subestimar o próprio consumo, não apresentando associação entre esta variável e a prevalência de sobrepeso ou obesidade. Outra explicação para ausência de associação é a causalidade reversa que está implícita aos estudos transversais quando exposições e desfechos são coletados simultaneamente 33. Dessa forma, este estudo recomenda preocupações metodológicas que levem em conta esses aspectos para o delineamento e execução de novos estudos.

Apesar da existência de algumas limitações, o estudo é inédito na região e representa o universo das escolas da zona urbana de Capão da Canoa. Seus resultados podem ser utilizados para desencadear uma série de medidas políticas e pedagógicas, úteis para o enfrentamento do problema da elevada prevalência de sobrepeso e obesidade e para evitar o agravamento do problema.
Os principais resultados deste estudo sugerem a necessidade ética do desencadeamento de ações voltadas para a promoção da saúde, possibilitando o envolvimento intersetorial 34 . Assim, os achados deste estudo também sugerem intervenções nas famílias quanto ao tempo de conduta sedentária, de hábitos familiares de alimentação e de atividade física. Em relação às escolas, a partir dos resultados pode-se inferir a importância da carga horária destinada para a prática de atividades físicas.

De qualquer forma as relações apresentadas nos parágrafos acima apontam para a seguinte hipótese: permanecer longos períodos em atividades sedentárias reduz o gasto energético com atividades físicas de lazer, o que por conseqüência desequilibra o balanço energético provocando o acúmulo de gordura corporal. Essa hipótese se fundamenta no estudo de Eaton et al. 35 , que sugerem uma redução histórica nos níveis de atividade física superior a uma redução no consumo energético apresentada na literatura por Prentice \& Jebb 36

A prevenção e o tratamento da obesidade devem, portanto, partir de uma completa modificação comportamental da sociedade, que propicie uma vida mais saudável, com uma alimentação de qualidade e atividade física preventiva. Além disso, ações públicas, que permitam o acesso de todos, independentemente da classe social 37 , a informações sobre os determinantes e conseqüências da obesidade são imprescindíveis para o controle da doença.

\section{Resumo}

Foi realizado um estudo transversal com o objetivo de determinar a prevalência de sobrepeso e obesidade e os fatores associados ao excesso de peso corporal, em um grupo de escolares de 11 a 13 anos, residentes em Capão da Canoa, Rio Grande do Sul, Brasil. A técnica de amostragem utilizada foi estratificada, proporcional ao número de alunos de cada escola. O estado nutricional foi avaliado mediante os pontos de corte do indice de massa corporal (IMC) de acordo com a idade e o sexo, propostos por Cole et al. no ano 2000. Foram analisadas variáveis sócio-econômicas, demográficas, genéticas e comportamentais. Foram entrevistados 719 escolares em 11 escolas, destes, 541 (75,2\%) apresenta- ram IMC considerado normal, 153 (21,3\%) escolares apresentaram sobrepeso e 25 (3,5\%) obesidade. Encontrou-se associação estatisticamente significativa com tipo de escola, estado nutricional dos pais, nível de atividade física e tempo de conduta sedentária. A prevalência de sobrepeso e obesidade encontrada nos escolares avaliados mostrou-se elevada (24,8\%), e, de acordo com a encontrada na literatura, confirmando a magnitude e gravidade que o problema assumiu entre os escolares de todo o Brasil.

Obesidade; Estado Nutricional; Exercício; Estudantes; Estudos Transversais 


\section{Colaboradores}

F. R. Suñé participou da coleta dos dados, da digitação das informações, da análise e redação do artigo. J. S. Dias-da-Costa, M. T. A. Olinto e M. P. Pattussi participaram da análise dos dados e da redação do artigo.

\section{Agradecimentos}

Artigo baseado na Dissertação de Mestrado, Prevalência de Sobrepeso e Obesidade em Escolares e Fatores Associados, de Fabio Rodrigo Suñé entregue para o Programa de Pós-graduação em Saúde Coletiva da Universidade do Vale do Rio dos Sinos em 2005.

\section{Referências}

1. World Health Organization. Obesity - preventing and managing the global epidemic. Geneva: World Health Organization; 1999. (WHO Technical Report Series, 894).

2. Gigante DP, Barros FC, Post CA, Olinto MTA. Prevalência de obesidade em adultos e seus fatores de risco. Rev Saúde Pública 1997; 31:236-46.

3. Instituto Brasileiro de Geografia e Estatística. Pesquisa de orçamentos familiares 2002-2003. http:// www.ibge.gov.br (acessado em 20/Jun/2006).

4. Instituto Brasileiro de Geografia e Estatística. Pesquisa de orçamentos familiares 2002-2003. Antropometria e análise do estado nutricional de crianças e adolescentes no Brasil. http://www.ibge.gov. br (acessado em 21/Nov/2006).

5. Albano RD, Souza SB. Estado nutricional de adolescentes: "risco de sobrepeso" e "sobrepeso" em uma escola pública do Município de São Paulo. Cad Saúde Pública 2001; 17:941-7.

6. Souza-Leão LSCD, Araújo LMB, Pimenta-de-Moraes LTL, Assis AM. Prevalência de obesidade em escolares de Salvador, Bahia. Arq Bras Endocrinol Metabol 2003; 47:151-7.

7. Wang Y, Monteiro C, Popkin BM. Trends of obesity and underweight in older children and adolescents in the United States, Brazil, China, and Russia. Am J Clin Nutr 2002; 75:971-7.

8. Veiga GV, Cunha AS, Sichieri R. Trends in overweight among adolescents living in the poorest and richest regions of Brazil. Am J Public Health 2004; 94:1544-8.
9. Bray GA. Sobrepeso, mortalidade e morbidade. In: Bouchard C, organizador. Atividade física e obesidade. São Paulo: Manole; 2003. p. 285-302.

10. Departamento de Informática do SUS. Mortalidade por causas associadas à obesidade no ano 2000. http://www.datasus.gov.br (acessado em 20/ Jun/2004).

11. Burrows R. Obesidad infantil y juvenil: consecuencias sobre la salud y la calidad de vida futura. Rev Chil Nutr 2000; 27:141-8.

12. Wright J, Pepe M, Seidel K, Dietz W, Whitaker R. Predicting obesity in young adulthood from childhood and parental obesity. N Engl J Med 1997; 337:869-73.

13. Kazapi IM, Di Pietro PF, Avancini SRP, Freitas SFT, Tramonte VLCG. Consumo de energia e macronutrientes por adolescentes de escolas públicas e privadas. Rev Nutr 2001; 14:27-33.

14. Fonseca VM, Sichieri R, Veiga GV. Fatores associados à obesidade em adolescentes. Rev Saúde Pública 1998; 32:541-9.

15. Gomes VB, Siqueira KS, Sichieri R. Atividade física em uma amostra probabilística da população do Município do Rio de Janeiro. Cad Saúde Pública 2001; 17:969-76.

16. Silva RCR, Malina RM. Nível da atividade física em adolescentes do Município de Niterói, Rio de Janeiro, Brasil. Cad Saúde Pública 2000; 16:1091-7.

17. Chiara VL, Sichieri R. Food consumption of adolescents. A simplified questionnaire for evaluating cardiovascular risk. Arq Bras Cardiol 2001; 77:337-41. 
18. Nahas MV. Atividade física, saúde e qualidade de vida: conceitos e sugestões para um estilo de vida ativo. 2a Ed. Londrina: Midiograf; 2001.

19. Cole TJ, Bellizze MC, Flegal KM, Dietz WH. Establishing a standard definition for child overweight and obesity worldwide: international survey. BMJ 2000; 320:1240-3.

20. Altman DG. Practical statistics for medical research. London: Chapman \& Hall; 1997.

21. Victora CG, Huttly SR, Fuchs SC, Olinto MT. The role of conceptual frameworks in epidemiological analysis: a hierarchical approach. Int J Epidemiol 1997; 26:224-47.

22. Giugliano R, Melo ALP. Diagnóstico de sobrepeso e obesidade em escolares: utilização do índice de massa corporal segundo padrão internacional. J Pediatr (Rio de J) 2004; 80:129-34.

23. Giugliano R, Carneiro EC. Factors associated with obesity in school children. J Pediatr (Rio de J) 2004; 80:17-22.

24. Sotelo YOM, Colugnati FAB, Taddei JAAC. Prevalência de sobrepeso e obesidade entre escolares da rede pública segundo três critérios de diagnóstico antropométrico. Cad Saúde Pública 2004; 20:233-40.

25. Victora CG, Vaughan JP, Barros FC, Silva AC, Tomasi E. Explaining trends in inequities: evidence from Brazilian child health studies. Lancet 2000; 356:1093-8.

26. Dias-da-Costa JS, Victora CG, Barros FC, Halpern R, Horta BL, Manzolli P. Assistência médica materno-infantil em duas coortes de base populacional no Sul do Brasil: tendências e diferenciais. Cad Saúde Pública 1996; 12 Suppl 1:59-66.

27. Carvalho CMRG, Nogueira AMT, Teles JBM, Paz SM, Sousa RML. Consumo alimentar de adolescentes matriculados em um colégio particular de Teresina, Piauí, Brasil. Rev Nutr 2001; 14:85-93.
28. Weineck J. Fundamentos gerais da biologia do esporte para a infância e adolescência. In: Weineck J, organizador. Biologia do esporte. São Paulo: Editora Manole; 1991. p. 246-64.

29. Ramos AMPP, Barros Filho AA. Prevalência da obesidade em adolescentes de Bragança Paulista e sua relação com a obesidade dos pais. Arq Bras Endocrinol Metabol 2003; 47:663-8.

30. Frutuoso MFP, Bismarck-Nasr EM, Gambardella AMD. Redução do dispêndio energético e excesso de peso corporal em adolescentes. Rev Nutr 2003; 16:257-63.

31. Jenovesi JF, Bracco MM, Colugnati FAB, Taddei JAAC. Perfil de atividade física em escolares da rede pública de diferentes estados nutricionais. Rev Bras Ciênc Mov 2003; 11:57-62.

32. Andrade RG, Pereira RA, Sichieri R. Consumo alimentar de adolescentes com e sem sobrepeso do Município do Rio de Janeiro. Cad Saúde Pública 2003; 19:1485-95.

33. Andrade A, Zicker F. Estudos de prevalência. In: Andrade A, Zicker F, organizadores. Métodos de investigação epidemiológica em doenças transmissíveis. Brasília: Organização Pan-Americana da Saúde/Fundação Nacional de Saúde; 1997. p. 33-42.

34. Campos GW, Barros RB, Castro AM. Avaliação da política nacional de promoção da saúde. Ciênc Saúde Coletiva 2004; 9:745-9.

35. Eaton SB, Cordain L, Gotshall RW. Physical activity, energy expenditure and fitness: an evolutionary perspective. Int J Sports Med 1998; 19:328-35.

36. Prentice AM, Jebb SA. Obesity in Britain: gluttony or sloth? BMJ 1995; 311:437-9.

37. Monteiro CA, Moura EC, Conde WL, Popkin BM. Socioeconomic status and obesity in adult populations of developing countries: a review. Public Health Rev 2004; 82:940-6.

Recebido em 13/Jul/2006

Versão final reapresentada em 04/Dez/2006

Aprovado em 09/Jan/2007 